\title{
Ablation of idiopathic ventricular arrhythmias originating from basal cardiac crux region
}

\author{
Pichmanil Khmao ${ }^{1}$, Chun Hwang ${ }^{2}$ and Hui-Nam Pak ${ }^{1 *}$ (1)
}

\begin{abstract}
Background: Ablation of idiopathic ventricular arrhythmias (VAs) in the cardiac crux region is one of the challenging procedures due to the complex anatomical structure where the four chambers of the heart are offset. Although this region is complex, the contiguous cardiac structures allow for the ablation of arrhythmias from adjacent sites.

Case presentation: We present different anatomical approaches in radiofrequency ablation and the ECG characteristics from a case series of VAs originated from the basal inferior ventricular septum, the corresponding endocardial aspect of the basal cardiac crux region.

Conclusions: Ablation of VAs originated from the basal cardiac crux region requires detailed mapping in the proximal coronary venous system and the adjacent structures including the RV, RA, and LV. In addition to the characteristic ECG of basal crux VAs, our three cases present an abrupt precordial transition in V2 with $\mathrm{R}$ wave amplitude greater than in $\mathrm{V} 1$ and $\mathrm{V} 3$.
\end{abstract}

Keywords: Cardiac crux region, Idiopathic ventricular arrhythmias, Radiofrequency ablation

\section{Introduction}

Idiopathic ventricular arrhythmias (VAs) occur in patients with a structurally normal heart. In most patients, these VAs originate from the outflow tract, while less common sites such as cardiac crux region are being identified based on their unique electrocardiographic (ECG) characteristics. Ablation of VAs in the cardiac crux region is a challenging procedure due to its anatomically complex structure, where the four chambers of the heart intersect. Although this region is complex, the contiguous cardiac structures allow for the ablation of these arrhythmias from adjacent sites. We present different anatomical approaches in RF ablation and the ECG characteristics of a case series of patients with VAs that

\footnotetext{
*Correspondence: hnpak@yuhs.ac

1 Yonsei University Health System, 50-1 Yonsei-ro, Seodaemun-gu, Seoul 03722, Republic of Korea

Full list of author information is available at the end of the article
}

originated from basal inferior ventricular septum, the corresponding endocardial aspect of basal cardiac crux region.

\section{Case 1}

The patient was a 21-year-old female who complained of palpitations and dizziness with chest discomfort and presented with monomorphic VT. Due to her unstable hemodynamic status, synchronized electrical cardioversion was delivered, which terminated the tachycardia and restored a normal sinus rhythm. There were no abnormalities on transthoracic echocardiography (TTE) and cardiac computed tomography $(\mathrm{CT})$ scan. The standard ECG obtained during VT (Fig. 1a) showed a left bundle branch block (LBBB) QRS morphology with a QS pattern in the leads II, III, and aVF. An rsR' complex in V2 with Rs wave at V5 and V6 was noted. Maximum deflection index (MDI) and pseudo-delta wave were 0.57 and $38 \mathrm{ms,}$ respectively. The patient underwent electrophysiological original author(s) and the source, provide a link to the Creative Commons licence, and indicate if changes were made. The images or other third party material in this article are included in the article's Creative Commons licence, unless indicated otherwise in a credit line to the material. If material is not included in the article's Creative Commons licence and your intended use is not permitted by statutory regulation or exceeds the permitted use, you will need to obtain permission directly from the copyright holder. To view a copy of this licence, visit http://creativecommons.org/licenses/by/4.0/. 
a

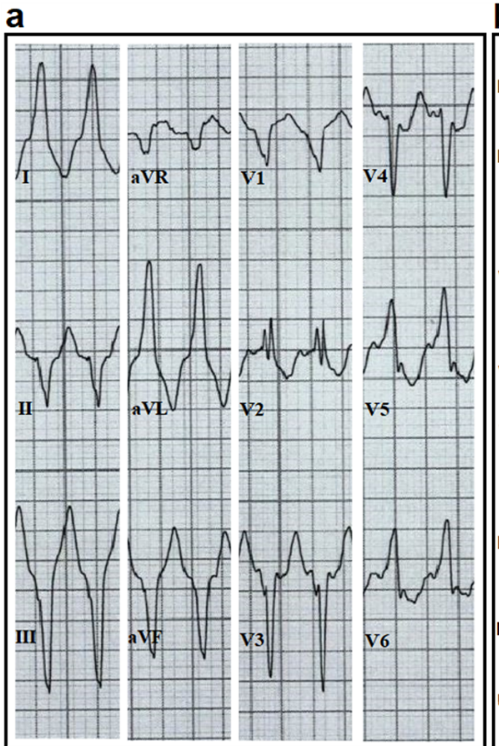

b

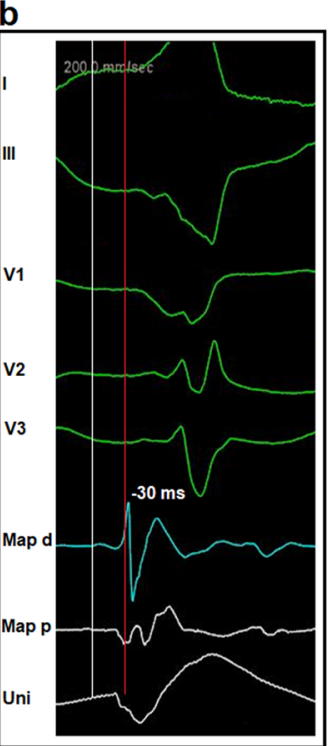

c

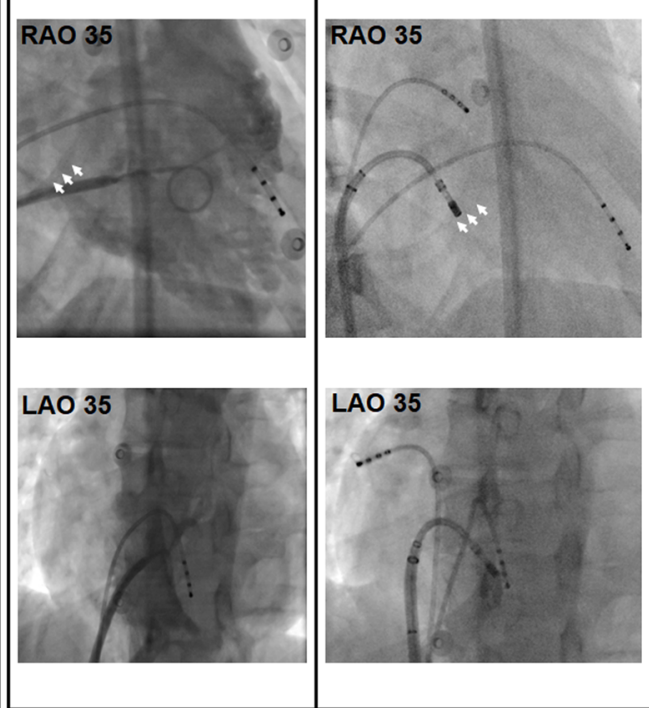

d

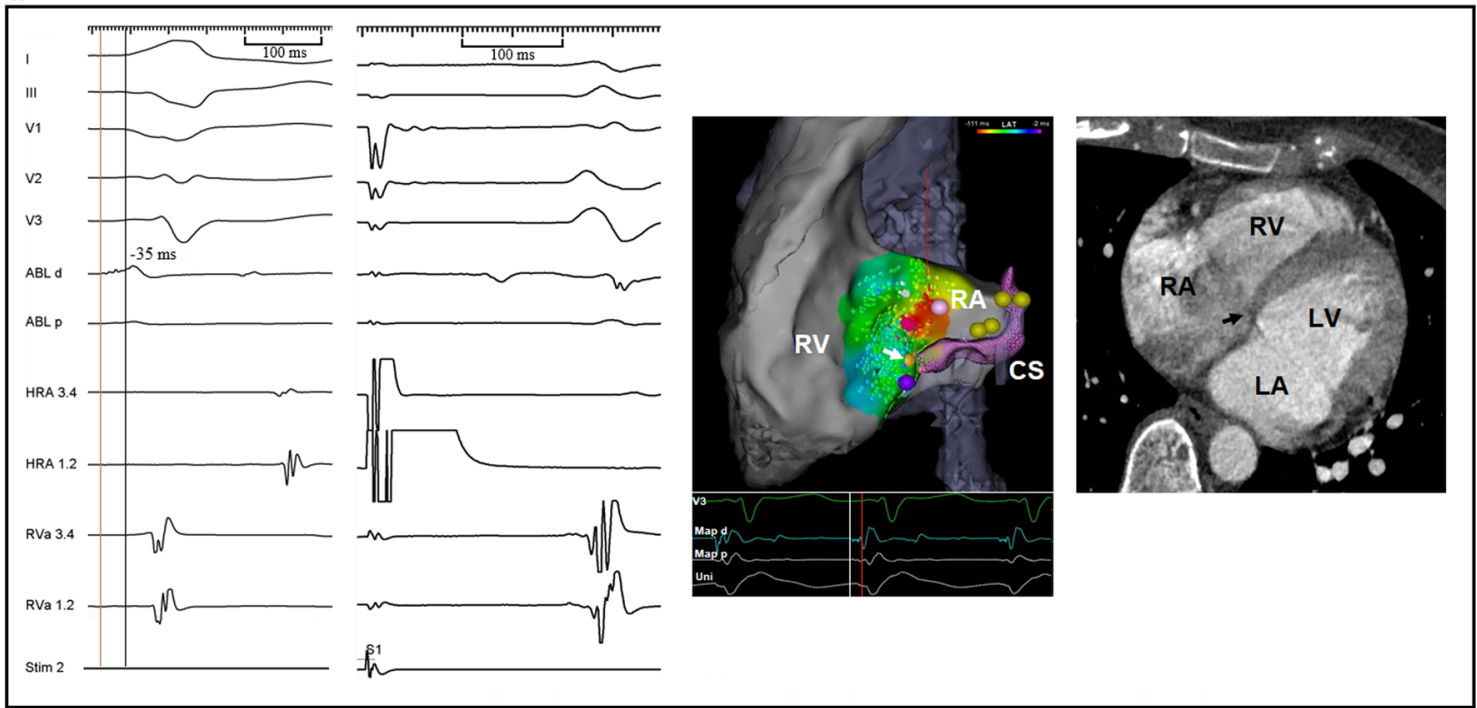

Fig. 1 a A 12-lead ECG of clinical VT. b Intracardiac EGM at the basal infero-septum of RV (the transient suppression site of VT). c Left panel: right ventriculography. Right panel: ablation catheter positioning at the successful site. $\mathbf{d}$ Left panel: intracardiac EGM at the successful ablation site during VT showed $V$ and $A$ signals at the distal ablation catheter. A and $V$ signals were also noted at the distal ablation catheter during atrial pacing. Middle panel: electro-anatomical mapping showed the successful ablation site (white arrow) from an RA approach close to the CS ostium (slow pathway region). Right panel: cardiac CT scan revealed the RA adjacent-side to the basal infero-septum of the LV (black arrow). CS indicates coronary sinus; EGM, electrogram; LAO, left anterior oblique view; LA, left atrium; $L V$, left ventricle; $R A$, right atrium; $R A O$, right anterior oblique view; RV, right ventricle

(EP) study, and clinical VT was induced by rapid right ventricular pacing. After right ventriculography (Fig. 1c, left panel), three-dimensional electro-anatomical mapping (CARTO, Biosense Webster, the USA) of the right ventricle (RV) was performed with a PentaRay (Biosense Webster, the USA) catheter during both normal sinus rhythm and VT. The location of the VT was precisely mapped, and the earliest activation sites (30 ms preQRS) were found at the basal infero-septum followed by ( $27 \mathrm{~ms}$ preQRS) the proximal of the middle cardiac vein (MCV). Despite a well-matched pace-mapping (PASO showed scoring of 98\%), the area of the earliest activation site was broad and the unipolar electrogram showed rS pattern at the target site (Fig. 1b). Radiofrequency energies (ThermoCool SmartTouch SF, Biosense Webster, the USA) were extensively delivered at the basal infero-septum 
from RV endocardium and resulted in only transient suppression of the clinical VT. Ablation within the proximal of MCV was limited by impedance rise. Alternative mapping at the right atrium (RA) adjacent-side to the basal infero-septum of the left ventricle (LV) was performed and showed a broad ventricular signal earlier than QRS onset by $35 \mathrm{~ms}$ followed by a local A signal at the distal mapping catheter (Fig. 1d left panel). Radiofrequency energies were applied with a power of $40 \mathrm{~W}$ and maximum temperature of $35{ }^{\circ} \mathrm{C}$ to the site (Fig. 1c, d) and resulted in complete elimination of VT without complications and were no longer inducible.

\section{Case 2}

A 58-year-old male patient presented a first episode of palpitations associated with chest discomfort after running a marathon and was diagnosed to have monomorphic VT. Transthoracic echocardiography was normal, and cardiac $\mathrm{CT}$ scan revealed non-significant coronary artery disease. Twelve-lead ECG obtained during VT (Fig. 2a) showed an LBBB morphology with rR' pattern in V2. A QS pattern in the inferior leads and an Rs wave in V5 were observed. An MDI and pseudo-delta wave were 0.65 and $60 \mathrm{~ms}$, respectively. The patient underwent EP study, and VT was induced by programmed ventricular extra-stimulus. A 2F octapolar JLL microcatheter via Judkins right 4 catheter (JR4) was placed through L1-type Swartz sheath (St. Jude Medical, St. Paul, MN, the USA), and mapping in the MCV where the proximal area showed local ventricular activation $35 \mathrm{~ms}$ earlier than QRS onset (Fig. 2b). Radiofrequency energies (ThermoCool SmartTouch SF, Biosense Webster, the USA) were delivered at the earliest ventricular site inside the proximal MCV but did not terminate the tachycardia. Further mapping was performed using PentaRay (Biosense Webster, the USA) catheter at basal infero-septum from the RV endocardium and showed earlier ventricular activation than the proximal MCV. The local ventricular electrogram preceded QRS onset by $38 \mathrm{~ms}$ at the site (Fig. 2c). Applications of RF energies with a power $40 \mathrm{~W}$ and maximum temperature of $35{ }^{\circ} \mathrm{C}$ at the target site eliminated VT without complications.

\section{Case 3}

A 77-year-old male patient who presented palpitations and exertional dyspnea was referred to our center because of non-sustained ventricular tachycardia (NSVT) and frequent premature ventricular complexes (PVCs). The patient had undergone percutaneous coronary intervention in the left anterior descending coronary artery one year prior to this admission. Transthoracic echocardiography revealed a reduced ejection fraction of $36 \%$ without significant LV chamber dilatation, suggesting potential PVC-induced cardiomyopathy. A 12-lead ECG of clinical PVC (Fig. 3a) revealed an LBBB pattern with abrupt precordial transition $\mathrm{R}$ wave in $\mathrm{V} 2$ and a $\mathrm{QS}$ pattern in the inferior leads. An MDI and pseudo-delta wave were 0.66 and $42 \mathrm{~ms}$, respectively. Three-dimensional electro-anatomical mapping (CARTO, Biosense Webster, CA, the USA) was performed on the RA and RV using a DecaNav catheter (Biosense Webster, the USA). An early ventricular activation of $33 \mathrm{~ms}$ earlier than QRS onset was seen in the infero-medial aspect of the RA which is anatomically adjacent to the basal inferior septum of the
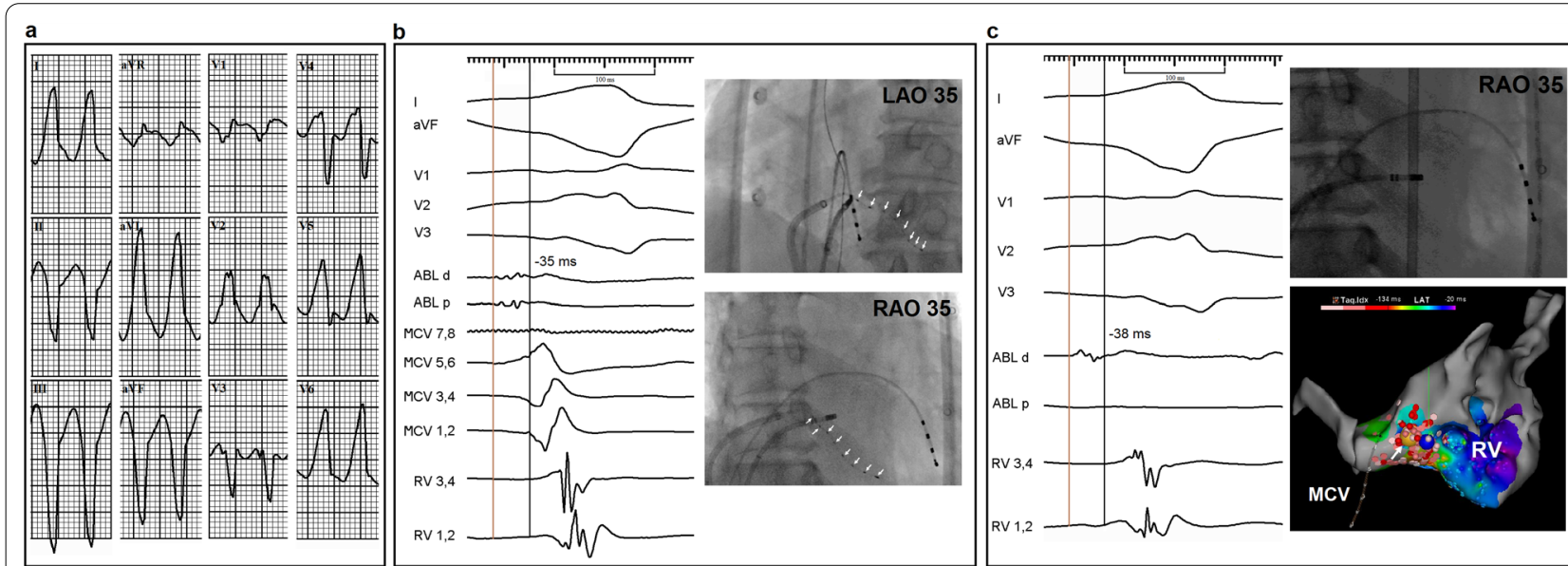

Fig. 2 a A 12-lead ECG of clinical VT. b Left panel: intracardiac EGM at the ablation site within the proximal of MCV. Right panel: ablation catheter positioning during the ablation within the proximal of MCV (small white arrows show the microcatheter positioned in MCV). $\mathbf{c}$ Left panel: intracardiac EGM at the successful ablation site showed the earliest ventricular activity of 38 ms preQRS. Right panel: ablation catheter positioning and electro-anatomical mapping showed the successful ablation site (white arrow) at the basal inferior ventricular septum. MCV indicates middle cardiac vein 


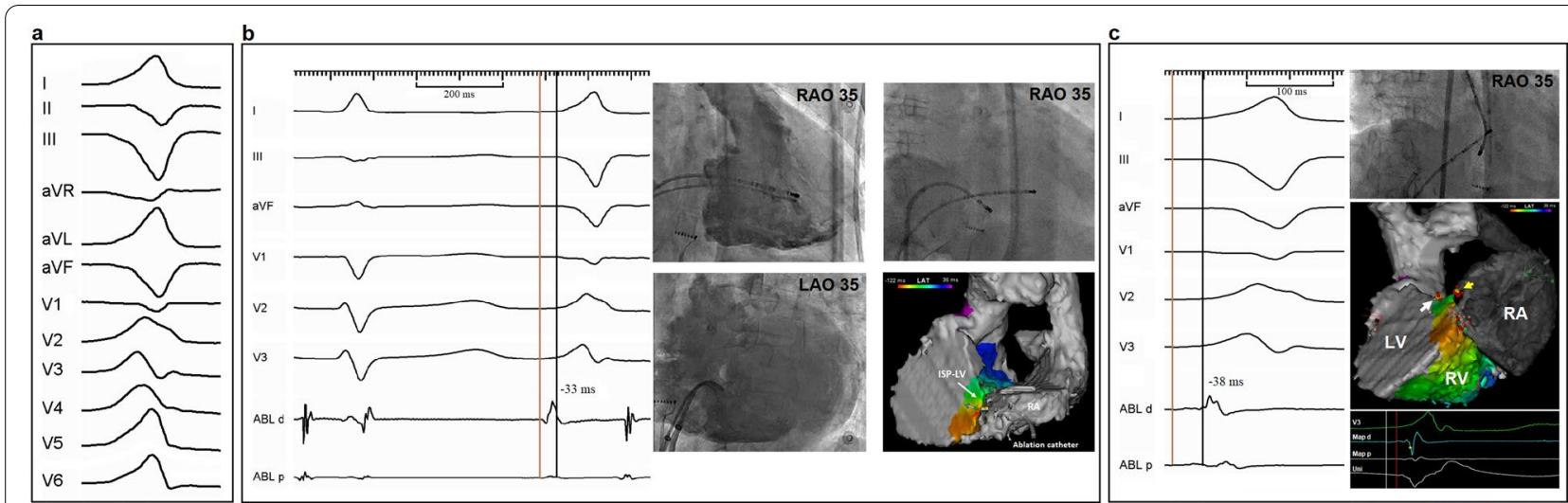

Fig. 3 a A 12-lead ECG of the clinical PVC. b Left panel: intracardiac EGM at the ablation site of the inferior septal process via an RA approach showed $V$ and $A$ signals. Middle panel: right ventriculography. Right panel: fluoroscopic view and electro-anatomical mapping with ablation catheter positioning via the RA infero-medial aspect, which is adjacent to inferior septal process. c Left panel: intracardiac EGM at the successful ablation site showed the earliest ventricular activity of $38 \mathrm{~ms}$ preQRS. Right panel: the application of RF energies at the inferior septal process via a retrograde trans-aortic approach. Note the far-field signal of PVC at the distal ablation might indicate intra-mural origin. Yellow arrow showed the ablation site via RA approach, and white arrow indicates the successful ablation site from retrograde approach. Note that, the sandwich lesion sets to completely eliminate the clinical PVC

LV (Fig. 3b). Applications of RF energies (ThermoCool SmartTouch SF, Biosense Webster, the USA) at the earliest ventricular activation site resulted in temporary suppression of the clinical PVC. A retrograde trans-aortic approach was performed to map the basal inferior septum from the LV endocardial aspect and revealed the earliest ventricular activation $38 \mathrm{~ms}$ preceded the QRS onset (Fig. 3c). Radiofrequency ablation was applied at a power of $50 \mathrm{~W}$ with maximum temperature $35^{\circ} \mathrm{C}$ at the latter site using a retrograde aortic approach and successfully ablated the clinical PVC.

\section{Discussion}

The cardiac crux region is a complex anatomical structure where the four chambers of the heart intersect. The inferior basal septum of the LV or the so-called inferior septal process (ISP) of the LV (attitudinally correct cardiac anatomy term) is a thick ventricular muscle located at the most basal inferior structure of the muscular interventricular septum, and it is one of the anteriorly boundary structures of the crux region. The surface ECG can suggest the epicardial origin of VAs from the basal crux region such as MDI $\geq 0.55$ and pseudo-delta wave duration $\geq 34 \mathrm{~ms}$ [1-3]. Arrhythmias that originate from the basal cardiac crux region are considered a subset of ISP arrhythmias, and RF ablation from the endocardial aspect of VAs that originate from the ISP has been recently reported $[4,5]$. Briceño et al. described the characteristics and ablation approach for idiopathic VAs that arise from the slow pathway region, which is a structure composed of the thin RA wall adjacent to the ISP [6]. Santangeli et al. described a catheter ablative technique with an infero-medial RA approach to eliminate VAs arising from the ISP after prior unsuccessful attempts [4]. As noted in the above discussion that the ISP is a thick muscular structure, Futyma et al. reported a case of ISP VT that was transiently suppressed using standard unipolar ablation and also required bipolar RF ablation delivered between the LV and RA sites to completely eliminate VAs [7]. A report from Kawamura et al. showed that the usual successful ablation site of the basal cardiac crux region is performed in the proximal of the MCV, while the apical crux VAs require percutaneous epicardial approach to completely eliminate the arrhythmias [8]. However, ablation within the proximal of the coronary venous system may increase the risk of procedural complications such as perforation and stenosis of coronary sinus as well as coronary artery damage including AV nodal and posterior descending arteries, which run through the pericrux area and close to the coronary venous branches. We experienced that ablation at the basal crux region requires extensive RF energy deliveries. Thus, recognizing the risk of procedural complications related to the ablation is crucial. Repeated sophisticated high-density mapping, careful power titration and using ablation index might be helpful for the safety procedure.

Interestingly, the above three cases present an abrupt precordial transition in $\mathrm{V} 2$ with $\mathrm{R}$ wave amplitude greater than in V1 and V3. However, the anatomical approach and the successful ablation sites were distinct in all three patients: on the RA adjacent-side to the ISP (Case 1), the basal infero-septum of the RV close to the ostium of MCV (Case 2), and the ISP from a retrograde aortic approach (Case 3). Anatomically, these target sites 
are contiguous structures in the basal crux area (Fig. 1d right panel) and therefore achieved the transient disappearance of VAs during extensive ablation at the adjacent structures. Therefore, the ablation of VAs at the basal crux region requires detailed mapping in the proximal coronary venous system as well as all the adjacent structures including the RV, RA, and LV. We also found that the contact force applied to the target site of the basal infero-septum of the RV was poor, and the ablation catheter could easily slip into the coronary sinus. In contrast, the contact force and catheter stability were better in the RA infero-medial approach.

\section{Conclusion}

Ablation of VAs originated from the basal cardiac crux region requires detailed mapping in the proximal coronary venous system and the adjacent structures including the RV, RA, and LV. The importance of understanding of the complex anatomical relationship within this region is crucial for successful ablation and avoiding complications. In addition, our three cases showed the characteristics ECG of basal crux VAs including a QS pattern in the inferior leads, an abrupt precordial transition in V2 with $\mathrm{R}$ wave amplitude greater than in $\mathrm{V} 1$ and $\mathrm{V} 3$ as well as an $\mathrm{R} / \mathrm{S}>1$ in $\mathrm{V} 5$ or $\mathrm{V} 6$.

\begin{abstract}
Abbreviations
ECG: Electrocardiogram; EGM: Electrogram; EP: Electrophysiology; CS: Coronary sinus; ISP: Inferior septal process; LBBB: Left bundle branch block; LV: Left ventricle; MCV: Middle cardiac vein; MDI: Maximum deflection index; NSVT: Non-sustained ventricular tachycardia; PVC: Premature ventricular complex; RA: Right atrium; RF: Radiofrequency; RV: Right ventricle; TTE: Transthoracic echocardiography; VA: Ventricular arrhythmia; VT: Ventricular tachycardia.
\end{abstract}

\section{Acknowledgements}

The authors thank Ms. Mingyeong Kim for the technical support with the CARTO images.

\section{Authors' contributions}

Pichmanil Khmao was a major contributor writing the manuscript. Chun Hwang and Hui-Nam Pak reviewed and revised the manuscript. All authors read and approved the final manuscript.

\section{Funding}

Not applicable.
Avaliability of data and materials

Not applicable.

\section{Declarations}

\section{Ethical approval and Consent to participate}

Written informed consents before procedure were given.

\section{Consent for publication \\ All data generated are anonymized.}

\section{Competing interests}

Authors declare no conflict of interests for this article.

\section{Author details}

${ }^{1}$ Yonsei University Health System, 50-1 Yonsei-ro, Seodaemun-gu, Seoul 03722, Republic of Korea. ${ }^{2}$ Department of Cardiology, Revere Health, Provo, UT, USA.

Received: 2 July 2021 Accepted: 2 August 2021

Published online: 22 September 2021

\section{References}

1. Berruezo A, Mont L, Nava S, Chueca E, Bartholomay E, Brugada J. Electrocardiographic recognition of the epicardial origin of ventricular tachycardias. Circulation. 2004;109:1842-7.

2. Daniels DV, Lu YY, Morton JB, et al. Idiopathic epicardial left ventricular tachycardia originating remote from the sinus of Valsalva: electrophysiological characteristics, catheter ablation, and identification from the 12-lead electrocardiogram. Circulation. 2006;113:1659-66.

3. Bazan V, Gerstenfeld EP, Garcia FC, et al. Site-specific twelve-lead ECG features to identify an epicardial origin for left ventricular tachycardia in the absence of myocardial infarction. Heart Rhythm. 2007;4:1403-10.

4. Santangeli P, Hutchinson MD, Supple GE, Callans DJ, Marchlinski FE, Garcia FC. Right atrial approach for ablation of ventricular arrhythmias arising from the left posterior-superior process of the left ventricle. Circ Arrhythm Electrophysiol 2016;9.

5. Li A, Zuberi Z, Bradfield JS, et al. Endocardial ablation of ventricular ectopic beats arising from the basal inferoseptal process of the left ventricle. Heart Rhythm. 2018;15:1356-62.

6. Briceno DF, Liang JJ, Shirai Y, et al. Clinical and electrophysiological characteristics of idiopathic ventricular arrhythmias originating from the slow pathway region. Heart Rhythm. 2019;16:1421-8.

7. Futyma P, Gluszczyk R, Futyma M, Kulakowski P. Right atrial position of a return electrode for bipolar ablation of the left posterosuperior process ventricular tachycardia. Pacing Clin Electrophysiol. 2019;42:474-7.

8. Kawamura M, Gerstenfeld EP, Vedantham V, et al. Idiopathic ventricular arrhythmia originating from the cardiac crux or inferior septum: epicardial idiopathic ventricular arrhythmia. Circ Arrhythm Electrophysiol. 2014;7:1152-8.

Ready to submit your research? Choose BMC and benefit from:

- fast, convenient online submission

- thorough peer review by experienced researchers in your field

- rapid publication on acceptance

- support for research data, including large and complex data types

- gold Open Access which fosters wider collaboration and increased citations

- maximum visibility for your research: over 100M website views per year

At BMC, research is always in progress.

Learn more biomedcentral.com/submissions 